\title{
La calidad de vida laboral y el soporte institucional para el trabajo en profesionales de las entidades públicas y privadas de Lima
}

\author{
The quality of working life and institutional support for work in \\ professionals of public and private entities in Lima
}

\author{
Alejandro E. Loli P. ${ }^{1}$ \\ Juan Danielli R. ${ }^{2}$ \\ Vladimir Navarro V. ${ }^{3}$ \\ Fernando Cerón V. ${ }^{4}$ \\ Universidad Nacional Mayor de San Marcos
}

Recibido: $18-04-18$

Aceptado: $23-07-18$

\section{Resumen}

La calidad de vida laboral es un constructo que ha demandado de muchos esfuerzos académicos y de investigación para delimitar sus alcances, especialmente en los países desarrollados. En el Perú, en cambio no se ha investigado mucho, menos sobre el rol del soporte institucional para el trabajo. Así, nuestro objetivo es conocer la relación entre calidad de vida laboral y el soporte institucional para el trabajo, en una muestra de 638 profesionales de organizaciones públicas y privadas que estudiaban maestrías, doctorados, segundas especialidades y diplomados de variadas especialidades en una universidad pública y otra privada, a quienes se le aplicó el cuestionario Calidad de Vida en el Trabajo (CVT GOHISALO), elaborado por González, Hidalgo, Salazar, Preciado (2010) en México. Nuestros hallazgos indican que existe insatisfacción en un alto porcentaje de profesionales tanto respecto de la calidad de vida laboral como del soporte institucional para el trabajo; así como hay una asociación de interdependencia entre ambas variables. Un análisis de estas variables con algunos factores socio demográficos y organizacionales nos permitió conocer las diferencias de opinión de los grupos con base en sus percepciones y experiencias laborales de los participantes.

Palabras clave: Calidad de vida laboral; soporte institucional; profesionales; entidades públicas y privadas; Perú.

\footnotetext{
1 Docente de la Universidad Nacional Mayor de San Marcos. Lima, Perú. Autor para correspondecia. Email: alolip@unmsm.edu.pe

2 Docente de la Universidad Nacional Mayor de San Marcos. Lima, Perú. Email: juan.danielli@unmsm.edu.pe 3 Docente de la Universidad Nacional Mayor de San Marcos. Lima, Perú. Email: oratoriateatro@hotmail.com 4 Docente de la Universidad Nacional Mayor de San Marcos. Lima, Perú. Email: fceronv@unmsm.edu.pe

(C) Los autores. Este artículo es publicado por la Revista de Investigación en Psicología de la Facultad de Psicología, Universidad Nacional Mayor de San Marcos. Este es un artículo de acceso abierto, distribuido bajo los términos de la licencia Creative Commons Atribucion - No Comercia_Compartir Igual 4.0 Internacional. (http://creativecommons.org/licenses/by-nc-sa/4.0/) que permite el uso no comercial, distribución y reproducción en cualquier medio, siempre que la obra original sea debidamente citada.
} 


\begin{abstract}
The quality of working life is a construct that has demanded many academic and research efforts to delimit its scope, especially in the developed countries. In Peru, however, much research has not been done, much less on the role of institutional support for work. Thus, our objective is to know the relationship between quality of work life and the institutional support for work. Using a sample of 638 professionals from public and private organizations in Lima and provinces, who studied masters, doctorates, second specialties and graduates of various specialties in a public and private university. Applying the questionnaire "Quality of Life at Work" (CVT GOHISALO) prepared by Raquel González-Baltazar, Gustavo Hidalgo-Santacruz, José G. Salazar Estrada, María L Preciado-Serrano (2010) in Mexico. Our findings indicate that there is dissatisfaction among a high percentage of professionals regarding both the quality of work life and institutional support for work; as well as there is an association of interdependence between both variables. An analysis of these variables with some socio-demographic and organizational factors allowed us to know the differences of opinion of the groups based on their perceptions and work experiences of the participants.

Keywords: Quality of working life; institutional support; professionals; public and private entities; Peru.
\end{abstract}

La población mundial crece en forma vertiginosa, las necesidades humanas son cada vez mayores y más exigentes; se rompen paradigmas a cada paso en todos los campos de la actividad social, política, económica, e incluso cultural. En las organizaciones productoras de bienes y servicios, igualmente, se crean, se ensayan e implementan nuevos modelos de gestión para ser más productivos, eficientes y eficaces a fin de encontrar el camino de solución a las necesidades apremiantes de la sociedad.

Así, el trabajo, en las organizaciones no solo constituyen la fuente principal de ingresos económicos de las personas, que les lleva a lograr satisfacciones materiales, sociales y psicológicas, sino también su permanencia por más de un tercio de su vida diaria, les permite desarrollarse en ellas como personas y cumplir su rol creativo en la sociedad.

Las organizaciones como ofertantes de fuentes de trabajo, están constituidas con una estructura definida, tienen objetivos claros, tienen una jerarquía y el trabajo está dividido de suerte que para el funcionamiento y cumplimiento de sus metas tienen que recurrir a personas a quienes se les asigna tareas, convirtiéndose en un lugar de convivencia humana, donde tienen que coordinar actividades e interactuar entre ellas (Schein, 1982). Entre tanto, si las condiciones que ofrece la organización les permiten a los trabajadores también oportunidades de desarrollo laboral y personal, y éstas le garantizan la satisfacción de sus necesidades de seguridad y crecimiento psicológico para su realización, los trabajadores percibirán mayor calidad de vida laboral, de caso contrario la percepción será diferente porque estas posibilidades no son comunes a todas las organizaciones (Peiró, 1993). Entonces, el soporte sostenido que puede dar la organización a sus colaboradores será de vital trascendencia. 
Si bien es cierto que la calidad de vida laboral, como concepto, tiene como antecedente más lejano a fines de la década de 1950 y durante los años de 1960, definiéndola como "reacción del individuo al trabajo o a las consecuencias personales de la experiencia laboral del trabajador" (Nadler y Lawler, 1983 pp.20-30, en Patlán, 2017), hoy en día y desde 1982, la calidad de vida laboral es concebida como una totalidad dentro de un enfoque integrador, convirtiéndose en una meta de la organización, "que se logra mediante procesos participativos y constituye una filosofía para las organizaciones" (Nadler y Lawler, 1983, en Peiró y Co, 1996, p. 167).

Dentro de la misma línea, Camacaro (2010, p 4), señala que la calidad de vida se puede considerar como una filosofía, esto es,

"...como un conjunto de creencias y valores que integran todos los esfuerzos dirigidos a incrementar la productividad y mejorar la moral de los trabajadores de la organización, haciendo énfasis en el reconocimiento de la dignidad del ser humano, así como su potencial intelectual, buscando incrementar la participación de las personas en el trabajo bajo un esquema continuo".

Sin duda que, en este caso, el ambiente laboral es fundamental para que los trabajadores se desarrollen, donde la relación de los gestores con los trabajadores sea más estrecha y directa para asegurar el éxito de los diferentes grupos de trabajo, generándose así, una autentica calidad de vida.

Drucker (1999), en cambio señala que la aplicación del conocimiento al trabajo para generar productividad e innovación, es lo más importante que podría otorgar la organización a sus trabajadores. Decía que, "si la empresa potencia a sus integrantes los estará haciendo empleables, es decir, capaces de proporcionarse por sí mismos condiciones que mejoren su calidad de vida" (en Camacaro, 2010 p.12). Es decir, darle oportunidad de desarrollo personal. Este es la tendencia de las organizaciones de los últimos años, pero muy pocas de ellas las asumen, y se resisten a seguir invirtiendo, porque muchos trabajadores reciben dicha formación y se retiran para integrarse a otras organizaciones que les ofrecen mejores condiciones de vida laboral. Claro está, que aquí la responsabilidad no es de aquel que se retira, tampoco de la organización que invierte, si no de los encargados de formular las estrategias a la culminación de aquella formación, a fin de retener ese capital humano. Vale decir, implementar apoyo institucional para mejorar su calidad de vida laboral.

Blanch (2002), cuando se refiere a la calidad de vida en el trabajo habla de satisfacción, de bienestar físico, psicológico y social que experimentan los trabajadoras en su centro de trabajo, y esto tiene que ver con las percepciones y valoraciones subjetivas y objetivas de los integrantes de la organización.

Sin embargo, la calidad de vida en el trabajo está determinada no solo por las características individuales (necesidades, valores, expectativas) o de situación 
(estructura organizacional, tecnología, sistema de remuneración, política interna) sino también por la actuación sistémica de estas características individuales y organizacionales (Bustamante, 2003, en Camacaro, 2010). Otros autores se refieren a calidad de vida profesional, relacionándolo al sentimiento de bienestar que surge entre las cargas de trabajo de la profesión y los recursos disponibles para afrontar esas exigencias (Clúa y Aguilar, 1998; Jubete, Lacalle, Riesgo, Cortés y Mateo, 2005), demostrando la evolución y del proceso integrador del constructo calidad de vida laboral y soporte institucional para el trabajo.

La complejidad del tema y la abundancia de definiciones sobre calidad de vida laboral, dice por sí mismo que no hay consenso entre los autores, y muchos estudiosos y con razón, suelen incorporar en sus informes la variedad de definiciones de dicho constructo. Otros, en cambio, han preferido agruparla en dos grandes formas de valorarla: la calidad psicológica de vida laboral y la calidad de vida objetiva. La primera, conocida también como subjetiva, que está relacionada con el bienestar y la salud de los trabajadores y, la segunda, relacionada con el entorno laboral, tales como las condiciones y el medio ambiente de trabajo (Rodríguez-Marín, 2010; Granados, 2011; Patlán, 2017).

Ahora bien, si existe alguna claridad en el constructo calidad de vida laboral, son las condiciones objetivas ofrecidas por la organización, como son el medio ambiente físico, tecnológico, contractual y productivo. En la medida en que todas ellas estén constituidas, institucionalizadas y sostenidas en el tiempo en armonía con las necesidades de la vida humana en la empresa, y cuyas consecuencias no causen riesgos en la salud física y psicológica de sus trabajadores, la calidad de vida laboral será satisfactoria o, en su caso, insatisfactoria; es decir, dependerá, en gran medida, del mayor o menor soporte que la organización le pueda dar a dichas condiciones, para que los integrantes de la organización cumplan su rol y garanticen el logro de las metas organizacionales. Como decía Prior y de la Poza, (1988), del soporte que la organización proporcione a sus trabajadores, dependerá la percepción y la experiencia que ellos tengan. Contrariamente, hay investigaciones que nos indican que, si bien existe una percepción positiva o favorable de la calidad de vida laboral, el apoyo directivo resulta la dimensión peor evaluada y la motivación intrínseca la mejor percibida por los funcionarios/ profesionales (Quezada, Sanhueza y Silva 2010; Calderón, Borracci, Sökn, Angel, Darú, Lerman y Trongé, 2014). Lo que podría significar que, a pesar de una percepción favorable y la motivación para el trabajo, si sienten que no hay soporte institucional, la situación podría variar en contra si la organización no actúa oportunamente.

La calidad de vida laboral subjetiva o psicológica, por su lado, abarca muchos factores. Por ejemplo, la relación entre la vida personal y familiar privadas de los trabajadores (necesidades de la familia) con las exigencias del trabajo y de la organización, pueden causar serias dificultades en ambos extremos: abandonar su 
familia por las responsabilidades asumidas en el trabajo, o alterar negativamente su desempeño y bajar su rendimiento laboral, en algunos casos, hasta abandonar sus tareas cotidianas (tardanzas, faltas, descansos médicos, etc.) por la responsabilidad de proteger a su familia; si la organización no le brinda un soporte sostenido para solucionar sus problemas familiares, ambas partes habrán perdido perspectiva, respecto de las metas organizacionales. Estudios empíricos demuestran que la calidad de vida laboral tiene una relación positiva y significativa con los resultados empresariales y del mismo modo influye en los resultados empresariales (Huerta, Pedraja, Contreras y Almodóvar, 2011).

Porotro lado, las personastienen el deseo de poneren pruebasus conocimientos, sus habilidades y destrezas e incluso sus actitudes y comportamiento en el desempeño de sus tareas cualquiera que fuera su profesión u ocupación; lo más importante para estas personas es su afán de logro, poder y afiliación para alcanzar la satisfacción de sus metas personales y encontrarle sentido al trabajo (McClelland, 1989; Peiró y Co, 1996); así como, la búsqueda de autonomía en la ejecución de sus tareas, la participación en la toma de decisiones y en la formulación de objetivos, que son la base de la satisfacción general con el trabajo (Casas y Co, 2002), son formas de expresión de su calidad de vida laboral; igualmente, la confianza, que es producto de las relaciones interpersonales que genera el apoyo mutuo entre compañeros de trabajo, mejorando los niveles de desempeño de los equipos de trabajo (Rodriguez y Co, 1997), la confianza asociada al empoderamiento de los profesionales en las organizaciones genera relaciones duraderas (Loli, 2016).

De allí que Ardila, (2003 p. 163), decía que,

“... calidad de vida es un estado de satisfacción general, derivado de la realización de las potencialidades de la persona. Posee aspectos subjetivos y aspectos objetivos. Es una sensación subjetiva de bienestar físico, psicológico y social. Incluye como aspectos subjetivos la intimidad, la expresión emocional, la seguridad percibida, la productividad personal y la salud objetiva. Como aspectos objetivos el bienestar material, las relaciones armónicas con el ambiente físico y social y con la comunidad, y la salud objetivamente percibida".

En el mismo sentido, Vergara, Alonso, Palacio y Rojas (2009) afirman que la calidad de vida laboral depende de la forma y condiciones de vida en las que una persona se desenvuelve, esto es, a la satisfacción experimentada por el trabajador respecto de esas condiciones objetivas y subjetivas que están relacionadas a la escala de valores, aspiraciones y expectativas personales.

Arias-Galicia (2000), considera que generar compromiso en las organizaciones incrementa la calidad de vida laboral y, el factor más importante para lograr el compromiso e incrementar la intención de permanencia, es el apoyo organizacional; o que la organización proporcione soporte a los empleados en situaciones de emergencia, y que se les considere como personas. 
De la misma manera, la seguridad, la integración al puesto de trabajo, la satisfacción por el trabajo, bienestar logrado a través del trabajo, el desarrollo personal y la administración del tiempo libre forman parte de ese proceso que determinan la calidad de vida laboral (González, Hidalgo, Salazar, y Preciado, 2010). No satisfacer estas variables, producen efectos colaterales relevantes (sobrecarga de trabajo, conflictos ético-profesionales, incertidumbre laboral, etc.) que deterioran la calidad de vida laboral y erosionan el servicio prestado, poniendo en cuestión la excelencia de la organización y algunos presuntos beneficios estratégicos (Blanch, 2014).

Aun cuando muchos autores consideran que calidad de vida laboral y soporte institucional para el trabajo son parte de un mismo constructo, probablemente porque encuentran asociación entre ellos, no significa que dicha relación sea causal; y, lo que se busca en el presente estudio es confirmar esa asociación, pero también determinar la probable influencia del soporte institucional para el trabajo en la calidad de vida laboral para el logro de las metas organizacionales, así como establecer las diferencias en el comportamiento de los participantes al intervenir las variables sociodemográficas.

\section{HIPÓTESIS}

Existe asociación entre el soporte institucional para el trabajo y la calidad de vida laboral de los profesionales de las entidades públicas y privadas de Lima.

\section{MÉTODO}

\section{Muestra:}

La muestra estuvo constituida por 638 profesionales que laboran como empleados o funcionarios de organizaciones públicas y privadas, y que cursan estudios de maestría, doctorado, segunda especialidad, o diplomaturas en las diferentes especialidades de una universidad pública y otra privada (educación, administración, negocios, sistemas e informática, contabilidad, psicología, tecnologías de información, project Management). Es una muestra intencional, no probabilístico debido a la variedad de profesiones, diversidad de procedencia y número de participantes de dichos grados académicos.

\section{Instrumento:}

El estudio se llevó a cabo con un cuestionario que lleva por nombre Calidad de Vida en el Trabajo (CVT GOHISALO), elaborado por González, Hidalgo, Salazar, Estrada, Preciado (2010). El cuestionario está constituido por siete dimensiones y un total de 74 ítemes con cinco (5) alternativas de respuesta y fue aplicado a 322 sujetos de los servicios de salud mexicanos, logrando una validez de $0.6777 \mathrm{y}$ una confiabilidad total de 0.9527 . Valores significativos que le dan estabilidad y confianza al instrumento. 
a. Confiabilidad del cuestionario en Perú

Para efectos de la presente investigación se procedió a una revisión de los ítemes por expertos, para determinar la validez de contenido, modificando algunas expresiones que, por razones de cultura, en una muestra peruana, pudiera no ser comprendido, manteniendo en esencia el sentido de las frases y oraciones y la estructura original. Luego, dicho instrumento se administró a 638 participantes de la muestra peruana y se sometió a análisis factorial exploratorio, obteniendo, de acuerdo al coeficiente alpha de Crombach, el valor total de 0.968 , mostrando estabilidad y confiabilidad de la prueba, como se puede ver en el cuadro que sigue.

\begin{tabular}{lc}
\hline Dimensiones & $\begin{array}{c}\text { Confiabilidad } \\
\text { Alfa de Cronbach }\end{array}$ \\
\hline Soporte institucional & .930 \\
Seguridad en el trabajo & .875 \\
Integración al puesto de trabajo & .826 \\
Satisfacción por el trabajo & .859 \\
Bienestar logrado a través del trabajo & .819 \\
Desarrollo personal & .851 \\
Administración del tiempo libre & .709 \\
\hline Confiabilidad total & .968 \\
\hline Confiabilidad del Cuestionario de Calidad de Vida en el Trabajo en una \\
muestra de trabajadores de entidades limeñas.
\end{tabular}

b. Validez de la escala

Posteriormente, se sometió a análisis factorial confirmatorio que mostró cargas factoriales en cada una de las dimensiones, resultando ser significativas $(\mathrm{Pr}=<0.05)$. En conclusión, los ítems están correlacionados con todos los componentes del instrumento, demostrando su capacidad de medir la Calidad de Vida en el Trabajo.

Como se puede apreciar, si se establece una comparación con los valores obtenidos en el análisis factorial exploratorio y confirmatorio original en muestra mexicana, se puede deducir que en la muestra peruana los valores son ligeramente mayores, probablemente debido a las diferencias culturales entre las muestras de los dos países. De manera que, el instrumento se mantiene con sus dimensiones, el número de ítemes y sus alternativas de respuesta para ser usado en el Perú sin alteraciones.

Finalmente, es preciso señalar que se incluyó, al cuestionario principal, datos adicionales relacionados a la información demográfica tales como: sexo, edad, grado de estudios concluidos, estado civil, años de servicio en la organización, años de servicio en el puesto, entidad o tipo de organización 
(pública o privada), lugar de procedencia, estatus ocupacional, lugar de residencia, remuneración bruta mensual, entre otros. Que servirán para ver las diferencias en la percepción de los participantes al intervenir las variables socio demográficas y organizacionales.

\section{Procesamiento de datos:}

Se utilizó el paquete estadístico SPSS (Statistical Package for the Social Sciences) para el tratamiento estadístico. De manera especial se utilizó el estadístico Alfa de Cronbach para conocer la confiabilidad y la validez del cuestionario, la correlación de Rho de Spearman para establecer la asociación entre los diferentes factores, y el Chi-Cuadrado para ver la percepción de los participantes sobre el soporte institucional y la calidad de vida laboral.

\section{RESULTADOS}

\section{Grado de satisfacción de los profesionales respecto de la calidad de vida laboral}

Una evaluación general (Tabla 1) de la percepción que tienen los profesionales de las organizaciones públicas y privadas de Lima, respecto de la Calidad de Vida Laboral, a través de los 7 componentes (Soporte institucional, Seguridad en el trabajo, Integración al puesto de trabajo, Satisfacción por el trabajo, Bienestar logrado a través del trabajo, Desarrollo personal, Administración del tiempo libre), a juzgar por la escala elaborada por quintiles, muestra que el 19.9\% está totalmente en desacuerdo con la calidad de vida que le ofrecen sus centros de trabajo, el $20.8 \%$ está en desacuerdo, el $20.8 \%$ es indiferente, solo el $18.7 \%$ está de acuerdo y el $19.7 \%$ está totalmente de acuerdo. Lo que significa que existe un alto porcentaje de profesionales insatisfechos con la calidad de vida que le brindan sus organizaciones, siendo éstos, mayor de aquellos que están satisfechos. Y, esta situación probablemente repercute, como es de esperarse, en el desempeño y en el rendimiento de los profesionales del sector público y privado de Lima.

Tabla 1

Calidad de vida laboral

\begin{tabular}{lccc}
\hline & Frecuencia & Porcentaje & $\begin{array}{c}\text { Porcentaje } \\
\text { acumulado }\end{array}$ \\
\hline Totalmente descuerdo & 127 & 19.9 & 19.9 \\
Desacuerdo & 133 & 20.8 & 40.8 \\
Indiferente & 133 & 20.8 & 61.6 \\
Acuerdo & 119 & 18.7 & 80.3 \\
Totalmente de acuerdo & 126 & 19.7 & 100.0 \\
\hline Total & 638 & 100.0 & \\
\hline
\end{tabular}




\section{Grado de soporte institucional para el trabajo y la calidad de vida laboral de los profesionales}

En la Tabla 2, se puede encontrar la relación de dependencia que existe entre la variable soporte institucional para el trabajo y la calidad de vida laboral total, donde el $14.8 \%$ de los participantes que están totalmente en desacuerdo con el soporte institucional, lo están también con la calidad de vida laboral, y 5.2\% en desacuerdo con la calidad de vida experimentado en su centro laboral. Entre tanto, aquellos que están simplemente en desacuerdo con el soporte institucional el $4.7 \%$ está totalmente en desacuerdo con la calidad de vida laboral y el 9.6\% expresa su indiferencia.

En el otro extremo, aquellos que dicen que están totalmente de acuerdo con el soporte o ayuda que reciben, el $15.1 \%$ se encuentra satisfecho con la calidad de vida que perciben y, el $2.8 \%$ está de acuerdo. Por otro lado, aquellos que dicen que están de acuerdo con la ayuda que reciben, el $4.1 \%$ está totalmente en desacuerdo con la calidad de vida que tienen, el $8.2 \%$ está de acuerdo y el $4.6 \%$ es indiferente a la calidad de vida recibida.

Finalmente, siendo el soporte institucional para el trabajo, una variable excepcionalmente importante para que los miembros de la organización tengan condiciones óptimas para desarrollar sus actividades y lograr sus metas personales, profesionales y organizacionales -a un grado de soporte institucional para el trabajo igual calidad de vida laboral- encontramos un alto porcentaje de profesionales que no están satisfechos con la calidad de vida que perciben o experimentan en su trabajo.

\section{Tabla 2}

Soporte institucional para el trabajo y calidad de vida laboral (muestra total)

\begin{tabular}{|c|c|c|c|c|c|c|c|c|}
\hline & & & \multicolumn{5}{|c|}{ Calidad de vida laboral total (agrupado) } & \multirow[b]{2}{*}{ Total } \\
\hline & & & $\begin{array}{l}\text { totalmente } \\
\text { descuerdo }\end{array}$ & desacuerdo & indiferente & acuerdo & $\begin{array}{l}\text { totalmente } \\
\text { de acuerdo }\end{array}$ & \\
\hline \multirow{10}{*}{$\begin{array}{l}\text { Soporte } \\
\text { institucional } \\
\text { (agrupado) }\end{array}$} & \multirow{2}{*}{$\begin{array}{l}\text { Totalmente } \\
\text { desacuerdo }\end{array}$} & Recuento & 94 & 33 & 5 & 0 & 0 & 132 \\
\hline & & $\%$ del total & $14.8 \%$ & $5.2 \%$ & $0.8 \%$ & $0.0 \%$ & $0.0 \%$ & $20.7 \%$ \\
\hline & \multirow{2}{*}{ Descuerdo } & Recuento & 30 & 61 & 43 & 11 & 0 & 145 \\
\hline & & $\%$ del total & $4.7 \%$ & $9.6 \%$ & $6.8 \%$ & $1.7 \%$ & $0.0 \%$ & $22.8 \%$ \\
\hline & \multirow{2}{*}{ Indiferente } & Recuento & 2 & 28 & 53 & 38 & 4 & 125 \\
\hline & & $\%$ del total & $0.3 \%$ & $4.4 \%$ & $8.3 \%$ & $6.0 \%$ & $0.6 \%$ & $19.6 \%$ \\
\hline & \multirow{2}{*}{ De acuerdo } & Recuento & 0 & 10 & 29 & 52 & 26 & 117 \\
\hline & & $\%$ del total & $0.0 \%$ & $1.6 \%$ & $4.6 \%$ & $8.2 \%$ & $4.1 \%$ & $18.4 \%$ \\
\hline & \multirow{2}{*}{$\begin{array}{l}\text { Totalmente de } \\
\text { acuerdo }\end{array}$} & Recuento & 0 & 1 & 3 & 18 & 96 & 118 \\
\hline & & $\%$ del total & $0.0 \%$ & $0.2 \%$ & $0.5 \%$ & $2.8 \%$ & $15.1 \%$ & $18.5 \%$ \\
\hline \multirow{2}{*}{ Total } & & Recuento & 126 & 133 & 133 & 119 & 126 & 637 \\
\hline & & $\%$ del total & $19.8 \%$ & $20.9 \%$ & $20.9 \%$ & $18.7 \%$ & $19.8 \%$ & $100.0 \%$ \\
\hline
\end{tabular}

$($ Chi-Cuadrado $=763.4, \mathrm{p}$-value $<.001)$ 
Por otro lado, de acuerdo al coeficiente de contingencia, esta asociación entre el soporte institucional para el trabajo y la calidad de vida laboral, es una fuerte relación de dependencia latente entre ambas variables. Que conduce a inferir la probabilidad de que hay influencia de la primera variable sobre la segunda.

\section{Asociación de dependencia}

\begin{tabular}{lccc}
\multicolumn{3}{c}{ Medidas simétricas } \\
\hline & Valor & Sig. aproximada \\
\hline Nominal por nominal & Coeficiente de contingencia & .738 & .000 \\
N de casos válidos & & 637 & \\
\hline
\end{tabular}

\section{Asociación de la calidad de vida laboral y el soporte institucional para el trabajo}

La Tabla 3, nos muestra el análisis correlacional entre el soporte institucional para el trabajo y la calidad de vida laboral de los profesionales de las organizaciones públicas y privadas de Lima, encontrándose que existe correlación muy significativa y positiva entre las dos variables $\left(\mathrm{r}=.884^{* *}\right)$. Confirmándose así, que a mayores condiciones laborales ofrecidas por la organización a sus profesionales (en procesos, en supervisión, evaluación del trabajo, oportunidades de promoción y mayor autonomía), éstos percibirán mejor calidad de vida laboral.

Tabla 3

Asociación de la calidad de vida laboral y el soporte institucional para el trabajo

\begin{tabular}{llcc}
\hline & & $\begin{array}{c}\text { Calidad de } \\
\text { vida total }\end{array}$ & Soporte institucional \\
\hline \multirow{3}{*}{ Calidad de vida total } & Coeficiente de correlación & 1.000 & $.884^{* *}$ \\
& Sig. (bilateral) & $\cdot$ & .000 \\
& $\mathrm{~N}$ & 638 & 637 \\
& Coeficiente de correlación & $.884^{* *}$ & 1.000 \\
soporte_institucional & Sig. (bilateral) & .000 &. \\
& $\mathrm{~N}$ & 637 & 637 \\
\hline
\end{tabular}

** La correlación es significativa al nivel 0.01 (bilateral).

Rho de Spearman

\section{La calidad de vida laboral y sus componentes}

En el análisis de correlación de los componentes de la calidad de vida laboral (Tabla 4) de los profesionales de entidades públicas y privadas de Lima se encontró que existe correlación muy significativa y positiva de seguridad en el trabajo con 
integración al puesto de trabajo $\left(\mathrm{r}=.642^{* *}\right)$, satisfacción por el trabajo $\left(\mathrm{r}=.666^{* *}\right)$, bienestar logrado a través del trabajo $\left(\mathrm{r}=.521^{* *}\right)$, desarrollo personal $\left(\mathrm{r}=.521^{* *}\right)$ y administración del tiempo libre $\left(\mathrm{r}=.460^{* *}\right)$; es decir, a mayor seguridad en el trabajo mayor integración al puesto de trabajo, mayor satisfacción por el trabajo, mayor bienestar logrado a través del trabajo, mayor desarrollo personal y mejor administración del tiempo libre.

También es significativa y positiva la correlación entre integración al puesto de trabajo y satisfacción por el trabajo $\left(\mathrm{r}=.719^{*}\right)$, bienestar logrado a través del trabajo $\left(\mathrm{r}=.731^{* *}\right)$, desarrollo personal $(\mathrm{r}=.731 * *)$ y administración del tiempo libre $\left(\mathrm{r}=.506^{* *}\right)$; luego, a mayor integración al puesto de trabajo mayor satisfacción por el trabajo, mayor bienestar logrado a través del trabajo, mayor desarrollo personal y mejor administración del tiempo libre. Igualmente, se halló una correlación significativa y positiva de satisfacción por el trabajo con bienestar logrado a través del trabajo $\left(\mathrm{r}=.672^{* *}\right)$, desarrollo personal $(\mathrm{r}=$ $.672 * *)$ y administración del tiempo libre $\left(\mathrm{r}=.530^{* *}\right)$; dicho de otro modo, a mayor satisfacción por el trabajo mayor bienestar logrado a través del trabajo, mayor desarrollo y mejor administración del tiempo libre. De la misma manera, se encontró correlación significativa y positiva de bienestar logrado a través del trabajo con desarrollo personal $\left(\mathrm{r}=1.000^{* *}\right)$ y administración del tiempo libre $\left(\mathrm{r}=.489^{* *}\right)$; lo que quiere decir, a mayor bienestar logrado a través del trabajo mayor desarrollo personal y mejor administración del tiempo libre. Finalmente, se encontró correlación significativa y positiva de desarrollo personal con administración del tiempo libre $\left(\mathrm{r}=.489^{* *}\right)$; lo que significa, a mayor desarrollo personal mejor administración del tiempo.

Por último, la tabla 4 muestra correlación significativa y positiva de soporte institucional para el trabajo con seguridad en el trabajo $\left(\mathrm{r}=.719^{* *}\right)$, integración al puesto de trabajo $\left(\mathrm{r}=.735^{* *}\right)$, satisfacción por el trabajo $(\mathrm{r}=$ $\left..668^{* *}\right)$, bienestar logrado a través del trabajo $\left(\mathrm{r}=.552^{* *}\right)$, desarrollo personal $\left(\mathrm{r}=.552^{* *}\right)$ y administración del tiempo libre $\left(\mathrm{r}=.476^{* *}\right)$; luego, mayor soporte institucional para el trabajo mayor seguridad en el trabajo, mayor integración al puesto de trabajo, mayor satisfacción por el trabajo, mayor bienestar logrado a través del trabajo, mayor desarrollo personal y mejor administración del tiempo libre.

A manera de conclusión, se puede decir que la asociación entre los componentes de la calidad de vida laboral es consistente y, el valor más alto, significativo y positivo es respecto del soporte institucional para el trabajo $(\mathrm{r}=$ $\left..884^{* *}\right)$; de allí que, se muestra no solo la asociación entre estas dos variables sino la influencia que puede estar ejerciendo el soporte institucional para el trabajo sobre la calidad de vida laboral de los profesionales de las organizaciones públicas y privadas, debido a esa fuerte relación de dependencia. 
Tabla 4

Correlación entre los componentes de calidad de vida laboral

\begin{tabular}{|c|c|c|c|c|c|c|c|c|c|}
\hline & & 1 & 2 & 3 & 4 & 5 & 6 & 7 & $\begin{array}{l}\text { Calidad } \\
\text { de vida }\end{array}$ \\
\hline \multirow{3}{*}{ 1. Seguridad en el trabajo } & Coeficiente $r$ & 1.000 & & & & & & & \\
\hline & Sig. (bilateral) & . & & & & & & & \\
\hline & $\mathrm{N}$ & 638 & & & & & & & \\
\hline \multirow{3}{*}{$\begin{array}{l}\text { 2. Integración al puesto de } \\
\text { trabajo }\end{array}$} & Coeficiente $r$ & $.642^{* *}$ & 1.000 & & & & & & \\
\hline & Sig. (bilateral) & .000 & . & & & & & & \\
\hline & $\mathrm{N}$ & 637 & 637 & & & & & & \\
\hline \multirow{3}{*}{ 3. Satisfacción por el trabajo } & Coeficiente $r$ & $.666^{* *}$ & $.719^{* *}$ & 1.000 & & & & & \\
\hline & Sig. (bilateral) & .000 & .000 & . & & & & & \\
\hline & $\mathrm{N}$ & 638 & 637 & 638 & & & & & \\
\hline \multirow{3}{*}{$\begin{array}{l}\text { 4. Bienestar logrado a través del } \\
\text { trabajo }\end{array}$} & Coeficiente $r$ & $.521^{* *}$ & $.731^{* *}$ & $.672^{* *}$ & 1.000 & & & & \\
\hline & Sig. (bilateral) & .000 & .000 & .000 & . & & & & \\
\hline & $\mathrm{N}$ & 638 & 637 & 638 & 638 & & & & \\
\hline \multirow{3}{*}{ 5. Desarrollo personal } & Coeficiente $r$ & $.521^{* *}$ & $.731^{* *}$ & $.672^{* *}$ & $1.000^{* *}$ & 1.000 & & & \\
\hline & Sig. (bilateral) & .000 & .000 & .000 & . & . & & & \\
\hline & $\mathrm{N}$ & 638 & 637 & 638 & 638 & 638 & & & \\
\hline \multirow{3}{*}{$\begin{array}{l}\text { 6. Administración del tiempo } \\
\text { libre }\end{array}$} & Coeficiente r & $.460^{* *}$ & $.506^{* *}$ & $.530^{* *}$ & $.489^{* *}$ & $.489^{* *}$ & 1.000 & & \\
\hline & Sig. (bilateral) & .000 & .000 & .000 & .000 & .000 & . & & \\
\hline & $\mathrm{N}$ & 638 & 637 & 638 & 638 & 638 & 638 & & \\
\hline \multirow{3}{*}{ 7. Soporte institucional } & Coeficiente $\mathrm{r}$ & $.719^{* *}$ & $.735^{* *}$ & $.668^{* *}$ & $.552^{* *}$ & $.552^{* *}$ & $.476^{* *}$ & 1.000 & \\
\hline & Sig. (bilateral) & .000 & .000 & .000 & .000 & .000 & .000 & . & \\
\hline & $\mathrm{N}$ & 637 & 636 & 637 & 637 & 637 & 637 & 637 & \\
\hline \multirow{3}{*}{ Total } & Coeficiente & $.850^{* *}$ & $.867^{* *}$ & $.845^{* * *}$ & $.755^{* *}$ & $.755^{* *}$ & $.621^{* *}$ & $.884^{* *}$ & 1.000 \\
\hline & Sig. (bilateral) & .000 & .000 & .000 & .000 & .000 & .000 & .000 & . \\
\hline & $\mathrm{N}$ & 638 & 637 & 638 & 638 & 638 & 638 & 637 & 638 \\
\hline
\end{tabular}

Rho de Spearman

**. La correlación es significativa al nivel .01 (bilateral).

\section{La relación de la calidad de vida laboral según las variables socio demográficas y organizacionales}

Un análisis de la calidad de vida laboral en relación con las variables socio demográficas y organizacionales, nos permitió conocer el comportamiento de los participantes agrupados en función de las características de la muestra. Las tablas correspondientes a 5.2 y siguientes se han omitido por razones de espacio para su publicación ( $\mathrm{Si}$ hubiera interés en conocer la totalidad de las tablas pueden solicitar al correo electrónico del autor principal). 


\subsection{Calidad de vida laboral según grado de instrucción concluido}

En la Tabla 5, se puede observar la relación de la calidad de vida laboral según el grado de instrucción concluido, siendo ésta significativa $(\mathrm{p}$-value $=$ .043).Aquellos que dicen tener una calidad de vida laboral baja el 61.5\% declaran tener estudios de educación superior universitaria, el 37.7\% dice tener estudios de maestría y $0.4 \%$ dice haber hecho estudios de doctorado. Por otro lado, aquellos que dicen que son indiferentes respecto a su calidad de vida laboral, el $63.2 \%$ dicen tener estudios de educación universitaria, 33.8\% dicen tener estudios de maestría y el $2.3 \%$ dice haber hecho estudios de doctorado. Entre tanto, aquellos que dicen tener alta calidad de vida laboral, el 54.3\% tiene educación superior universitaria, el $44.5 \%$ tiene estudios de maestría y el 1.2\% dice haber realizado otro tipo de estudios.

En conclusión, se puede decir que existe diferencia de opinión entre los profesionales de educación superior universitaria, maestría y doctorado, predominando la indiferencia entre los que tienen educación superior universitaria y doctorado respecto de la calidad de vida laboral, en cambio los que tienen maestría consideran que tienen una calidad de vida laboral alta.

Tabla 5

Calidad de vida laboral según grado de instrucción concluido

\begin{tabular}{llccccc}
\hline & & \multicolumn{2}{c}{ GRADO INSTRUCCIÓN CONCLUIDO } & Total \\
\cline { 3 - 5 } & & $\begin{array}{c}\text { Superior } \\
\text { universitaria }\end{array}$ & Maestría & Doctorado & Otro & \\
\hline \multirow{2}{*}{ Bajo } & Recuento & 160 & 98 & 1 & 1 & 260 \\
& \% dentro de reca_total_2 & $61.5 \%$ & $37.7 \%$ & $0.4 \%$ & $0.4 \%$ & $100.0 \%$ \\
\multirow{2}{*}{ Indiferente } & Recuento & 84 & 45 & 3 & 1 & 133 \\
\cline { 2 - 5 } & \% dentro de reca_total_2 & $63.2 \%$ & $33.8 \%$ & $2.3 \%$ & $0.8 \%$ & $100.0 \%$ \\
\hline \multirow{2}{*}{ Total } & Recuento & 133 & 109 & 0 & 3 & 245 \\
& \% dentro de reca_total_2 & $54.3 \%$ & $44.5 \%$ & $0.0 \%$ & $1.2 \%$ & $100.0 \%$ \\
\hline
\end{tabular}

p-value $=.043$

5.2. Calidad de vida laboral según la entidad y/o empresa a la que pertenecen

Se puede observar la relación de la calidad de vida laboral según la entidad y/o empresa a las que pertenecen, siendo ésta significativa ( $p$-value $=.004)$. Los profesionales que señalan que tienen una calidad de vida laboral baja, el 54.2\% pertenecen a organizaciones privadas y el $44.6 \%$ pertenecen a entidades públicas. Del mismo modo, los profesionales que son indiferentes respecto de la calidad de vida laboral, el 55.6\% pertenecen a organizaciones privadas, el $43.6 \%$ a entidades 
públicas. Entre tanto, aquellos profesionales que señalan que tienen una alta calidad de vida profesional, el $67.3 \%$ pertenecen a organizaciones privadas y el $29.8 \%$ a entidades públicas.

De lo señalado se podría deducir, que existe diferencia de opinión de los profesionales que laboran en las organizaciones privadas de aquellos que laboran en las entidades públicas respecto de la calidad de vida laboral, siendo baja en las organizaciones públicas y alta en las organizaciones privadas.

\subsection{Calidad de vida laboral según estatus ocupacional}

Se muestra la relación de la calidad de vida laboral según el estatus ocupacional que ostentan, siendo ésta significativa ( $\mathrm{p}$-value $=, 017$ ). Los profesionales que dicen tener calidad de vida laboral baja, el $88.5 \%$ son empleados y el $4.6 \%$ son directivos y el $6.9 \%$ tienen un estatus diferente. Aquellos que son indiferentes respecto de la calidad de vida laboral, el $82.7 \%$ son empleados, el $7.5 \%$ son directivos y el $9.8 \%$ son de otro tipo de estatus. Mientras que los que tienen un estatus ocupacional alto, el $80.4 \%$ son empleados, el $12.7 \%$ son directivos y el $6.9 \%$ son otro tipo de estatus.

A manera de conclusión, la calidad de vida laboral baja predomina entre los empleados y alta entre los directivos, los que tienen un estatus ocupacional distinto son indiferentes respecto a la calidad de vida laboral. Es decir, hay diferencia de opiniones.

\subsection{Calidad de vida laboral según remuneración bruta mensual}

Encontramos la relación de la calidad de vida laboral según remuneración bruta mensual que perciben, siendo ésta significativa ( $p$-value $=.001$ ). Aquellos profesionales que señalan que tienen una calidad de vida baja, el $34.6 \%$ dice ganar una remuneración que va de 850 a 2000 soles mensuales, el $21.9 \%$ gana entre 2001 a 3000, 18.1\% gana entre 3001 a 4000 y, el 25.4\% gana más de 4001 soles. Los profesionales que son indiferentes respecto de la calidad de vida laboral, el $32.3 \%$ tienen una remuneración de 850 a 2000 soles mensual, el $18.8 \%$ tiene un ingreso mensual de 2001 a 3000 soles, el $23.3 \%$ tiene un ingreso mensual de 3001 a 4000 soles y, el $25.6 \%$ tiene un ingreso mensual igual o mayor a 4001 soles. Mientras que los profesionales que señalan que tienen una calidad de vida laboral alta, el $18.8 \%$ dice tener un ingreso mensual entre 850 y 2000 , el $16.3 \%$ indica que sus ingresos mensuales se encuentran entre 2001 y 3000 , el $20.4 \%$ dice ganar mensualmente entre 3001 y 4000 y, el $44.5 \%$ señala que tiene una remuneración igual o mayor a 4001 soles.

Aquí, se puede inferir que los profesionales que tienen una remuneración bruta mensual de 850 a 2000 y de 2001 a 3000 tienen una calidad de vida laboral baja, mientras que los que tienen un ingreso bruto mensual de 3001 a 4000 son 
indiferentes respecto de la calidad de vida laboral, y aquellos que tienen una remuneración igual o mayor a 4001 tienen una calidad de vida laboral alta. Dicho de otro modo, hay diferencia de opinión.

\subsection{Calidad de vida laboral según lugar de residencia}

Se muestra la relación de la calidad de vida laboral según el lugar de residencia de los profesionales. Siendo ésta significativa ( $p$-value $=.042$ ). Aquí, los profesionales que consideran que calidad de vida laboral es baja, el $94.2 \%$ residen en Lima y el $5.8 \%$ en provincia. Aquellos que dicen ser indiferentes respecto de la calidad de vida laboral, el $89.5 \%$ residen el Lima, mientras que el 10.5\% dicen residir en provincia. Entre tanto, aquellos profesionales que indican que tienen alta calidad de vida laboral, el 95.95 reside en Lima y el $4.1 \%$ vive en provincia.

En conclusión, hay diferencias de opinión entre aquellos que residen en Lima y los que residen en provincias, predominando la calidad de vida laboral alta en Lima, e indiferente en provincias.

Finalmente, es necesario precisar que las variables socio demográficas y organizacionales, tales como el género, la edad, el estado civil, el lugar de procedencia, los años de servicio en la organización y los años de servicio en el puesto de trabajo no resultaron significativas. Lo que podría estar indicándonos que estas variables no cambian la opinión de los profesionales de las entidades públicas y privadas y son homogéneos respecto de la calidad de vida laboral.

\section{Relación del soporte institucional para el trabajo según las variables socio demográficas y organizacionales}

El análisis de resultados sobre el soporte institucional para el trabajo (Procesos de trabajo, Supervisión laboral, Apoyo de los superiores para la realización del trabajo, Evaluación del trabajo, Oportunidades de promoción y Autonomía) otorgado a los profesionales de las entidades públicas y privadas, serán tratadas en este acápite en relación con los grupos socio demográficas y organizacionales.

6.1. Soporte institucional para el trabajo según los grupos etáreos de los participantes

Se puede observar la relación del soporte institucional para el trabajo según los grupos etáreos, siendo ésta significativa ( $\mathrm{p}$-value $=.048$ ). Los profesionales que consideran que el soporte institucional recibido es bajo, el $34.7 \%$ son de 20 a 30 años, el $41 \%$ son de 31 a 40 años, $13.4 \%$ de 41 a 50 y, de $10.1 \%$ son sujetos mayores de 51 años. Aquellos que son indiferentes respecto del soporte institucional que proporciona la organización, el $37.6 \%$ son de 20 a 30 años, el $44.8 \%$ son de 31 a 40 años, el $16.0 \%$ son de 41 a 50 años, el $1.6 \%$ son iguales o mayores de 51 
años. Mientras que los profesionales de dicen que tienen soporte institucional alto brindada por sus centros de trabajo, el $37.4 \%$ son de 20 a 30 años, el $38.7 \%$ son de 31 a 40 años, el $15.3 \%$ son de 41 a 50 años y el $8.5 \%$ son iguales o mayores a 51 años de edad.

En conclusión, existe diferencia de opinión según la edad; se puede inferir que a opinión de los profesionales de 20 a 30, de 31 a 40 y de 41 a 50 años de edad el apoyo institucional para el trabajo es indiferente en comparación al apoyo alto y bajo; mientras que en opinión de aquellos mayores a 51 años de edad el apoyo institucional para el trabajo es bajo.

6.2. El soporte institucional para el trabajo según el tipo de organización a la cual pertenecen los participantes

Se puede observar la relación del soporte institucional para el trabajo según el tipo de organización de donde proceden los profesionales, siendo ésta significativa $(\mathrm{p}$-value $=.002)$. Aquellos profesionales que consideran el soporte institucional que reciben como bajo, el $45.8 \%$ son de entidades públicas, el 52.7\% son de entidades privadas y el $1.4 \%$ son de otro tipo de organización. Los que ven con indiferencia el soporte institucional que brinda la organización, el 37.6\% son de entidades públicas, y el $62.4 \%$ son de organizaciones privadas. Mientras que aquellos profesionales que consideran que el apoyo institucional que reciben es alto, el $31.1 \%$ son de entidades públicas, el $66.0 \%$ son de empresas privadas y, el $3.0 \%$ son de otros tipos de organización.

De la descripción previa se puede concluir, que los profesionales que laboran en entidades públicas tienen un bajo soporte institucional para el trabajo, mientras que los profesionales que laboran en organizaciones privadas mayor soporte institucional para el trabajo, al igual que aquellos que laboran en otro tipo de instituciones; entonces hay diferencias de opinión entre estos grupos.

6.3. Soporte institucional según el estatus ocupacional que ostentan los participantes

Se puede observar la relación del soporte institucional para el trabajo según el estatus ocupacional de los profesionales, siendo ésta significativa $(\mathrm{p}$-value $=$ .013). Los profesionales que dicen tener un bajo soporte institucional, el $86.6 \%$ son empleados, el $4.7 \%$ son directivos y el $8.7 \%$ son los que se califican de otro estatus.

Entre tanto, los que son indiferentes al apoyo que reciben de la organización, el $85.6 \%$ son empleados, $7.2 \%$ son directivos y el otro $7.2 \%$ son de otro estatus. Mientras que, los que consideran que tiene un soporte institucional alto, el $80.4 \%$ son empleados, el $13.2 \%$ son directivos y el $6.4 \%$ son de otro estatus. 
Así se puede concluir, que los profesionales no directivos tienen un bajo soporte institucional para el trabajo, al igual que aquellos que son de otro tipo de estatus; mientras que los directivos tienen un soporte institucional alto para el trabajo; por tanto, hay diferencia por estatus ocupacional. mensual.

6.4. El soporte institucional para el trabajo según la remuneración bruta

Se puede apreciar la relación del soporte institucional para el trabajo según la remuneración bruta mensual de los profesionales, siendo ésta significativa ( $\mathrm{p}$-value $=.001)$. Los profesionales que consideran que el soporte institucional recibido es bajo, el $33.9 \%$ son los que ganan de 850 a 2000 soles, el $22.4 \%$ son los tienen como ingresos de 2001 a 3000 soles, el $21.7 \%$ son los que tienen como ingreso de 3001 a 4000 soles, y el $22.0 \%$ son los que tienen como ingreso igual o mayor a 4001soles. Aquellos que son indiferentes al apoyo que reciben de su organización, el 24\% son los que ganan de 850 a 2000, el $19.2 \%$ son los que ganan de 2001 a 3000 soles, el $16.8 \%$ son los que ganan de 3001 a 4000 soles, y el $40.0 \%$ son los que tienen como ingreso mensual, igual o mayor de 4001 soles. Entre tanto, los profesionales que consideran que tienen un alto soporte institucional, el $23.0 \%$ ganan de 850 a 2000 soles, el $15.3 \%$ son los que tienen ingresos de 2001 a 3000 soles, el $20 \%$ son aquellos que ganan de 3001 a 4000 soles, el $41.7 \%$ son los tienen como ingreso mensual igual o mayor a 4001 soles.

En conclusión, existe diferencia significativa de opinión en aquellos profesionales que tienen una remuneración que van entre 850 a 4000, dicen tener un soporte institucional para el trabajo bajo, mientras que los profesionales que tienen una remuneración igual o superior a 4001 dicen tener un soporte institucional para el trabajo alto.

Finalmente, resulta necesario señalar que las demás variables sociodemográficas y organizacionales, tales como género, estado civil, grado de instrucción, lugar de procedencia, años de servicio en la organización, años de servicio en el puesto y lugar de residencia no han resultado significativas, permitiéndonos deducir que dichas variables no repercuten en la percepción o experiencia que el profesional pueda tener respecto del apoyo que les brinda su organización. En decir, son homogéneos.

\section{DISCUSIÓN}

En términos generales, los resultados de la presente investigación nos muestran que existe un alto porcentaje de profesionales insatisfechos con la calidad de vida que le brindan sus organizaciones, siendo éste, mayor que aquellos que están satisfechos. En el lenguaje de Blanch, (2002), solo una parte menor de los profesionales habrían logrado experimentar satisfacción y bienestar físico, 
psicológico y social en su lugar de trabajo, sin duda con base en sus percepciones y valoraciones subjetivas y objetivas. Aunque existen autores que indican que estas características van más allá de las individuales y que tiene que ver con un comportamiento sistémico porque incluye las características de la organización (Bustamante, 2003, en Camacaro, 2010). De allí la importancia de poner énfasis en las condiciones estructurales, psicológicas y sociales que ofrecen las organizaciones para una mejor calidad de vida.

Las consecuencias, como es natural, repercutirán en el desempeño y en el rendimiento de los profesionales del sector público y privado de Lima; afectando el incremento de la productividad como decía Camacaro (2010, p. 4), quien ponía especial atención, también “... en mejorar la moral de los trabajadores de la organización, haciendo énfasis en el reconocimiento de la dignidad del ser humano". En efecto, el soporte institucional para el trabajo, juega aquí un papel importante para que los miembros de la organización tengan condiciones óptimas para desarrollar sus actividades y lograr sus metas personales, profesionales y organizacionales. Otros autores se refieren a calidad de vida profesional, relacionándolo al sentimiento de bienestar que surge entre las cargas de trabajo de la profesión y los recursos disponibles para afrontar esas exigencias (Clúa, y Aguilar, 1998; Jubete, Lacalle, Riesgo, Cortés y Mateo, 2005). Por eso, como país estamos aún lejos de que nuestras organizaciones tengan como meta de la organización mejorar la calidad de vida laboral en el corto plazo, incluso sabiendo que se puede lograr mediante procesos participativos (Patlán, 2017; Camacaro, 2010), tanto en la toma de decisiones, en la formulación de objetivos, como en la búsqueda de autonomía en la ejecución de sus tareas, que son la base de la satisfacción general con el trabajo (Casas y Co, 2002).

Por otro lado, nuestros hallazgos indican que, entre el soporte institucional para el trabajo y la calidad de vida laboral, existe una fuerte relación de interdependencia entre ambas variables. De hecho, este resultado demuestra la razón por la que otros autores lo consideren como una dimensión más de la calidad de vida laboral (González, Hidalgo, Salazar y Preciado, 2010); sin embargo, no es posible una calidad de vida laboral sostenida -acaso una influencia sobre la calidad de vida laboral- sin el soporte que la organización pueda proporcionar, porque la percepción y la experiencia de los integrantes de la organización dependen de ese apoyo (Prior y de la Poza, 1988).

Estos conceptos armonizan con nuestras pesquisas en el sentido que, cuanto mayores condiciones laborales ofrece la organización a sus profesionales (en procesos, en supervisión, valoración del trabajo, oportunidades de promoción y mayor autonomía), éstos percibirán mejor la calidad de vida psicológica en el trabajo. A este último se le llama también subjetiva, que está relacionada con el bienestar y la salud de los trabajadores y, calidad de vida objetiva, relacionada 
con el entorno laboral, tales como las condiciones y el medio ambiente de trabajo (Rodríguez, 2010; Granados, 2011; Patlán, 2017).

Un análisis más detallado sobre los componentes de la calidad de vida laboral nos demuestra que hay asociación entre todos ellos; demostrando consistencia del instrumento, pero, sobre todo, la asociación del soporte institucional para el trabajo con la calidad de vida laboral, que tiene el puntaje mejor valorado y positivo $\left(\mathrm{r}=.884^{* *}\right)$ en opinión de los participantes. Cuando se habla de calidad de vida laboral nos referimos a procesos, derechos contractuales, salarios, capacitación, motivación, ambiente de trabajo, participación, reconocimiento, logros, expectativas, manejo del tiempo libre, intimidad, expresión emocional, seguridad percibida (Ardila, 2003) entre otros ; por consiguiente, una planificación bien diseñada y las buenas prácticas, puede llevar al logro de las metas organizacionales; sin embargo, aun cuando la percepción fuera favorable y positiva sobre la calidad de vida, muchos de estos factores pueden resultar divergentes y alterar el conjunto de factores. Por ejemplo, una investigación demuestra que el apoyo directivo resulta la dimensión peor evaluada y la motivación intrínseca la mejor percibida por los funcionarios/profesionales (Quezada, Sanhueza, y Silva, 2010; Calderón, Borracci, Sökn, Angel, Darú, Lerman, y Trongé, 2014). De allí que el soporte institucional para el trabajo adquiera mayor relevancia. Pero desde el otro extremo, como decía Blanch (2014), no satisfacer estas variables, producen efectos colaterales relevantes (sobrecarga de trabajo, conflictos ético-profesionales, incertidumbre laboral, etc.) que deterioran la calidad de vida laboral y erosionan el servicio prestado, poniendo en cuestión la excelencia de la organización y algunos presuntos beneficios estratégicos.

Un análisis de la relación calidad de vida laboral con las variables socio demográficas y organizacionales, nos indica la diferencia de opinión de los participantes de acuerdo al grado de instrucción obtenido, tipo de organización donde trabajan, estatus ocupacional, la remuneración que persiguen y el lugar de residencia. Destacando la diferencia de opinión de los profesionales que laboran en organizaciones públicas y privadas, predominando la calidad de vida laboral baja en las públicas y alta en las privadas.

De la misma manera, se realizó un análisis sobre la diferencia de opinión respecto del soporte o apoyo institucional para el trabajo otorgado pororganizaciones en relación con las variables socio demográficas y organizacionales, hallando dicha diferencia de acuerdo a la edad, tipo de organización, estatus ocupacional y la remuneración bruta mensual que perciben. Entre ellos, se podría señalar la opinión diferenciada por el tipo de entidad donde laboran, cuya opinión al apoyo recibido en entidades privadas es más favorable en comparación con las públicas que es más desfavorable. 
La relación de la calidad de vida laboral y el soporte institucional para el trabajo con otras variables socio demográficas y organizacionales no señaladas antes, resultaron siendo variables no diferenciadoras; por tanto, son homogéneas y las opiniones no varían con su intervención.

Finalmente, es pertinente señalar que la presente investigación demanda aún de otros esfuerzos, tal como la influencia de la calidad de vida laboral con el desempeño, a fin de confirmar las afirmaciones hechas en este estudio, pero, sin duda, también será necesario con otras variables en sectores similares al presente.

\section{CONCLUSIONES}

1. En términos generales se puede afirmar que existe un alto porcentaje de profesionales insatisfechos con la calidad de vida laboral que le brindan sus organizaciones, siendo éstos mayor de aquellos que están satisfechos. Situación que probablemente puede estar repercutiendo en el desempeño y en el rendimiento de los profesionales del sector público y privado de Lima.

2. Existe asociación entre el soporte institucional para el trabajo y la calidad de vida laboral, es una fuerte relación de dependencia entre ambas variables. Es decir, a una posición de desacuerdo respecto de la ayuda institucional para el trabajo corresponde igual posición respecto de la calidad de vida laboral; lo mismo ocurre con una postura de estar de acuerdo. En otros términos, hay una interdependencia entre ambas variables. Así, cuanto mayores condiciones laborales ofrece la organización a sus profesionales (en procesos, en supervisión, evaluación del trabajo, oportunidades de promoción y mayor autonomía y otros), éstos percibirán mejor calidad de vida laboral.

3. Un análisis de correlaciones entre los componentes de la calidad de vida laboral nos muestra asociación consistente y, el valor más alto, significativo y positivo es respecto del soporte institucional para el trabajo $\left(\mathrm{r}=.884^{* *}\right)$; de allí que, se muestra no solo la asociación entre estas dos variables sino la influencia que puede estar ejerciendo el soporte institucional para el trabajo sobre la calidad de vida laboral de los profesionales.

4. Un análisis sobre la relación de la calidad de vida laboral con las variables socio demográficas y organizacionales arroja los siguientes hallazgos:

a. Existe diferencia de opinión entre los profesionales de educación superior universitaria, maestría y doctorado, predominando la indiferencia entre los que tienen educación superior universitaria y doctorado respecto de la calidad de vida laboral, en cambio los que 
tienen maestría consideran que tienen una calidad de vida laboral alta.

b. Existe diferencia de opinión de los profesionales que laboran en las organizaciones privadas de aquellos que laboran en las entidades públicas respecto de la calidad de vida laboral, siendo baja en las organizaciones públicas y alta en las organizaciones privadas.

c. La calidad de vida laboral baja predomina entre los empleados y alta entre los directivos, los que tienen un estatus ocupacional distinto son indiferentes respecto a la calidad de vida laboral. Es decir, hay diferencia de opiniones.

d. Los profesionales que tienen una remuneración bruta mensual de 850 a 3000 soles dicen tener una calidad de vida laboral baja, mientras que los que tienen un ingreso bruto mensual de 3001 a 4000 soles son indiferentes respecto de la calidad de vida laboral, y aquellos que tienen una remuneración igual o mayor a 4001 soles tienen una calidad de vida laboral alta. Dicho de otro modo, hay diferencia de opinión.

e. Hay diferencias de opinión entre aquellos que residen en Lima y los que residen en provincias, predominando la calidad de vida laboral alta en Lima, e indiferente en provincias.

5. Un análisis sobre la relación del soporte institucional para el trabajo con las variables socio demográficas y organizacionales arroja que:

a. Existe diferencia de opinión de los profesionales de 20 a 50 años de edad respecto del soporte institucional para el trabajo, siendo estos de opinión indiferente en comparación al apoyo alto y bajo; mientras que en opinión de aquellos mayores a 51 años de edad respecto del apoyo institucional para el trabajo es bajo.

b. Los profesionales que laboran en entidades públicas opinan que tienen un bajo soporte institucional para el trabajo, mientras que los profesionales que laboran en organizaciones privadas dicen tener mayor soporte institucional para el trabajo, al igual que aquellos que laboran en otro tipo de instituciones; estableciéndose la diferencia de opinión entre estos grupos.

c. Los profesionales no directivos (empleados) opinan que tienen un bajo soporte institucional para el trabajo, al igual que aquellos que son de otro tipo de estatus; mientras que los directivos dicen tener soporte institucional alto para el trabajo; por tanto, hay diferencia por estatus ocupacional. 
d. Los profesionales que tienen una remuneración que van entre 850 a 4000 soles, dicen tener un soporte institucional para el trabajo bajo, mientras que los profesionales que tienen una remuneración igual o superior a 4001 soles dicen tener un soporte institucional para el trabajo alto.

\section{RECOMENDACIONES}

1. Las instituciones del sector público, demandan de una decisión política organizacional para comprometerse en el diseño, promoción e implementación de programas de intervención a fin de mejorar las condiciones que dan soporte institucional a la calidad de vida laboral.

2. Las organizaciones del sector privado que, si bien cuentan con un porcentaje mayor de satisfacción de sus profesionales respecto de la calidad de vida laboral, es pertinente seguir promoviendo programas de intervención que eleven mejores niveles de satisfacción reforzando las condiciones de apoyo institucional.

\section{REFERENCIAS BIBLIOGRÁFICAS}

Ardila, R., (2003). Calidad de vida: una definición integradora. Colombia:Fundación Universitaria Konrad Lorenz. Revista Latinoamericana de Psicología, Vol. 35, $\mathrm{N}^{\circ} 2$, pp 161-164. Recuperado: http://www.redalyc.org/pdf/805/80535203.pdf

Arias-Galicia, F. (2000, Octubre). El compromiso personal hacia la organizacional y la intención de permanencia: Factores para su incremento. México, UNAM. Recuperado: http://www.ejournal.unam.mx/rca/200/RCA20001.pdf

Blanch, J. (2002). Teoría de las relaciones laborales. España: Ed. UOC.

Blanch, J. (2014). Calidad de vida laboral en hospitales y universidades mercantilizados. España: Universitat Autónoma de Barcelona. Rev. Papeles del Psicólogo, Vol. $35 \mathrm{~N}^{\circ}$ 1, pp 40-47. Recuperado:http://www.papelesdelpsicologo.es/pdf/1164.pdf

Calderón, J; Borracci, R.; Sökn, F.; Angel, A.; Darú, V.; Lerman, J. y Trongé J., (2014, Octubre). Encuesta sobre la calidad de vida profesional de los cardiólogos en la Argentina. Argentina: Revista argentina de cardiología. Vol. 82, $\mathrm{N}^{\circ}$ 5, 389-395. Recuperado: https://www.researchgate.net/publication/287353461

Camacaro, P. (2010, Marzo).Abordaje conceptual de calidad de vida en el trabajo. Venezuela: Universidad Nacional Experimental de la Fuerza Armada. Recuperado: https://www.researchgate.net/publication/46562438

Casas J. y Co (2002, Julio/Setiembre). Dimensiones y medición de la calidad de vida laboral en profesionales sanitarios. Revista de administración sanitaria, Vol. VI, N . 23, pp 143-160. 
Clúa JL. y Aguilar, C. (1998, Setiembre). La calidad de vida profesional y el orgullo de trabajar en la sanidad pública. España: Sociedad española de familia y comunitaria. Vol. 22 N 5 , pp308-314. Rescatado: https:/dialnet.unirioja.es/servlet/ articulo?codigo $=2892789$

Drucker, P. (2002). La Sociedad Post Capitalista, (2da Edición). Bogotá: Ed. Norma.

Drucker, P. (2002). Los desafíos de la gerencia para el siglo XXI. Bogotá: Ed. Norma.

González-Baltazar, R.; Hidalgo-Santacruz,G.; Salazar, J. y Preciado-Serrano, M. (2010, Abril/Junio). Elaboración y validez del instrumento para medir calidad de vida en el trabajo "cvt-gohisalo" Ciencia \& Trabajo Vol. 12 N $^{\circ} 36$, pp 332-340. Recuperado: Available at: http://works.bepress.com/jose_salazar/4/).

Granados, I. (2011, Diciembre). Calidad de vida laboral: historia, dimensiones y beneficios. Lima: Revista de Investigación en Psicología, V. 14, º2, pp271-276. UNMSM.

Huerta, P., Pedraja, L., Contreras, S. y Almodóvar, P. (2011, Octubre-Diciembre). Calidad de vida laboral y su influencia sobre los resultados empresariales. Chile: Revista de Ciencias sociales, vol. 17, N 4, 658 - 676. Recuperado: http://produccioncientificaluz. org/index.php/rcs/article/view/13841/13824

Jubete MT, Lacalle M, Riesgo R, Cortés JA, Mateo C. (2005, Junio). Estudio de la calidad de vida profesional en los trabajadores de atención primaria del Área 1 de Madrid. Aten Primaria. España: Vol.36, N² pp 112-114. Recuperado: https://www. sciencedirect.com/science/article/pii/S0212656705704418

Patlan, J. (2017). Significado psicológico del constructo "calidad de vida” en el trabajo mediante redes semánticas naturales. México: Universidad Autónoma del Estado de Morelos.

Peiró, J.M. (1993). Desencadenantes del estrés laboral. Madrid: Eudema.

Peiró, J. y Co.(1996). Tratado de psicología del trabajo. Madrid: Síntesis.

Prior, J. y de la Poza, J. (1988, Marzo) Calidad de vida en el trabajo: un estudio empírico. España: I Congreso Iberoamericano de psicología del trabajo y de las organizaciones. Recuperado: https://dialnet.unirioja.es/servlet/articulo?codigo=1250544

Quezada, F.; Sanhueza, A. y Silva F. (2010, Mayo). Diagnóstico de la calidad de vida laboral percibida por los trabajadores de cuatro servicios clínicos del complejo asistencial "Dr. Víctor Ríos Ruiz" de los ángeles. Colombia: Universidad Biobio, Rev. Horizontes empresariales, Vol. 9, $\mathrm{N}^{\circ}$ 1, pp 55-68. Recuperado: http://revistas. ubiobio.cl/index.php/HHEE/article/view/2037

Rodriguez, JM y Co. (1997). El reto del trabajo en equipo. Biblioteca IESE de Gestión de Empresas.Barcelona: Ediciones Folio S.A.

Rodríguez-Marín, J., (2010). Calidad de vida laboral en profesionales de la salud. España: Rev. Calidad Asistencial. Vol. $25 \mathrm{~N}^{\circ}$ 6, 318-320. Departamento de Psicología de la Salud, Universidad Miguel Hernández, Elche, Alicante. Recuperado: https://dialnet. unirioja.es/servlet/articulo?codigo $=3394768$ 
Schein, E. (1982). Psicología de la Organización. Madrid: Ed. Dossat S.A.

Vergara D., R., Alonso, L., Palacio, J.E. y Rojas, M. (2009, Abril/Junio). El desarrollo humano en la calidad de vidas integradas en un modelo de gestión urbana para Barranquilla. Colombia: Revista Salud Uninorte Vol. 25, N 2, 374-390. Recuperado: http://ciruelo.uninorte.edu.co/pdf/salud_uninorte/25-2/14_\%20El\%20 Desarrollo\%20Humano\%20y\%201a\%20Calidad $\% 20$ de $\% 20$ Vida.pdf 